\title{
Composite materials based on dental acrylic plastic and chitosan
}

\author{
L.A. Yakovishin * (D), E.V. Tkachenko (iD
}

Sevastopol State University, 299053 University st., 33, Sevastopol, Russia

* Corresponding authors: chemsevntu@rambler.ru

This short communication (letter) belongs to the MOSM2O21 Special Issue.

(C) 2021, The Authors. This article is published in open access form under the terms and conditions of the Creative Commons Attribution (CC BY) license (http://creativecommons.org/licenses/by/4.o/).

\begin{abstract}
Chitosan and poly(methyl methacrylate) (PMMA) composites were synthesized by polymerization with heating and mechanochemical method. The obtained polymer composites were analyzed by the ATR FT-IR spectroscopy method. The presence of intermolecular hydrogen bonds and hydrophobic interactions in formation of PMMA and chitosan polymer composites was shown.
\end{abstract}

\author{
Keywords \\ polymer composites \\ poly(methyl methacrylate) \\ chitosan \\ FT-IR spectroscopy \\ Received: 03.11.2021 \\ Revised: 16.12 .2021 \\ Accepted: 16.12.2021 \\ Available online: 17.12.2021
}

\section{Introduction}

New composites are currently being actively studied [1-3]. Such materials may be promising for use in various fields of medicine. For example, composite materials are widely used in modern dentistry [1].

Poly(methyl methacrylate) or poly(methyl 2-methylpropenoate) (PMMA) is one of the most important polymers in industry and medicine $[1,4]$. PMMA is widely used in dentistry practice for the fabrication of dentures [4]. On the basis of PMMA, heat-curing acrylic resins for removable prostheses, cold-curing resins for frameworks and self-curing resins for denture repairs and for custom trays have been developed [5, 6]. However, PMMA can have a toxic effect on the human body, which is accompanied by allergic reactions and impaired oral microflora [7].

Polysaccharide chitosan is used for obtaining new biomedical materials $[8,9]$. Chitosan has antitumoral, antioxidative, bacteriostatic and fungistatic properties [8]. Earlier, various composites of chitosan with gelatin [10], clay [9] and other substances were prepared. In addition, a block copolymer of chitosan with PMMA was obtained [11]. This article is devoted to the preparation of composite materials based on PMMA and chitosan.

\section{Experimental}

Low-molecular water-soluble chitosan (freeze-dried powder; purity $88.4 \%$; molecular weight $1-30 \mathrm{kDa}$ ) was pur- chased from Bioprogress (Russia). The source of PMMA was the Villacryl H Plus (Zhermack S.p.A., Poland).

\subsection{Preparation of composites}

Preparation of composite $1.1 \mathrm{~g}$ of chitosan and $1 \mathrm{~g}$ of Villacryl $\mathrm{H}$ Plus were stirred for $1 \mathrm{~min}, 0.44 \mathrm{ml}$ of PMMA monomer was added to the resulting mixture, stirred for $10 \mathrm{~min}$ at room temperature, and heated at $60{ }^{\circ} \mathrm{C}$ for $60 \mathrm{~min}$.

Preparation of composite 2. $1 \mathrm{~g}$ of chitosan and $1 \mathrm{~g}$ of Villacryl $\mathrm{H}$ Plus were stirred for $1 \mathrm{~min}, 0.44 \mathrm{ml}$ of PMMA monomer was added to the resulting mixture, stirred for $10 \mathrm{~min}$ at room temperature and heated at $60{ }^{\circ} \mathrm{C}$ for $30 \mathrm{~min}$. Then the temperature was raised to $100{ }^{\circ} \mathrm{C}$ and the mixture was heated for another $30 \mathrm{~min}$.

Preparation of composite 3. $1 \mathrm{~g}$ of chitosan and $1 \mathrm{~g}$ of Villacryl $\mathrm{H}$ Plus were grinded in an agate mortar for $120 \mathrm{~min}$. To the resulting mixture was added $0.44 \mathrm{ml}$ of PMMA monomer, stirred for $10 \mathrm{~min}$ at room temperature and heated at $60^{\circ} \mathrm{C}$ for $30 \mathrm{~min}$. Then the temperature was raised to $100{ }^{\circ} \mathrm{C}$ and the mixture was heated for another $30 \mathrm{~min}$.

The powders of the obtained composites were cooled to room temperature and investigated by the FT-IR spectroscopy method. The IR spectra were recorded on the Simex FT801 IR-Fourier spectrometer (Russia) in the $4000-550 \mathrm{~cm}^{-1}$ region (spectral resolution $4 \mathrm{~cm}^{-1} ; 25$ scans) using the ATR accessory with diamante crystal plate. Photos of the composites were obtained using the ATR accessory with builtin lens (Simex, Russia) at 6ox magnification. 


\subsection{IR spectra}

IR spectrum of PMMA $\left(v, \mathrm{~cm}^{-1}\right): 2998(\mathrm{CH}), 2948(\mathrm{CH})$, $2853(\mathrm{CH}), 1719(\mathrm{C}=\mathrm{O}), 1474(\mathrm{CH}), 1458(\mathrm{CH}), 1432(\mathrm{CH})$, $1387(\mathrm{CH}), 1364(\mathrm{CH}), 1322(\mathrm{CH}), 1268$ (C-O-C), 1237 (C-O-C), 1188 (CO-O- $\left.\mathrm{CH}_{3}, \mathrm{CH}\right), 1140$ (C-O-C), $1059(\mathrm{C}-\mathrm{O}-\mathrm{C}), 984(\mathrm{C}-\mathrm{C}), 962(\mathrm{C}-\mathrm{C}), 912(\mathrm{CH}), 839(\mathrm{CH})$, $809(\mathrm{C}=\mathrm{O}), 747(\mathrm{C}=\mathrm{O}, \mathrm{CH}), 692(\mathrm{CH})$.

IR spectrum of chitosan $\left(v, \mathrm{~cm}^{-1}\right): 3310(\mathrm{OH}, \mathrm{NH})$, $2869(\mathrm{CH}), 2836(\mathrm{CH}), 1655\left(\mathrm{C}=\mathrm{O}_{\mathrm{Amid}}\right), 1619\left(\mathrm{NH}_{2}\right)$, $1609\left(\mathrm{NH}_{2}\right), 1602 \quad\left(\mathrm{NH}_{2}\right), 1543 \quad\left(\mathrm{~N}-\mathrm{H}+\mathrm{C}-\mathrm{N}_{\mathrm{Amid}} \mathrm{II}\right)$, $1516 \quad\left(\mathrm{~N}-\mathrm{H}+\mathrm{C}-\mathrm{N}_{\text {Amid II }}\right), \quad 1509 \quad\left(\mathrm{~N}-\mathrm{H}+\mathrm{C}-\mathrm{N}_{\text {Amid II }}\right)$, $1501\left(\mathrm{~N}-\mathrm{H}+\mathrm{C}-\mathrm{N}_{\mathrm{Amid}} \mathrm{II}\right), 1489(\mathrm{CH}), 1474(\mathrm{CH}), 1418(\mathrm{CH})$, $1379(\mathrm{CH}), \quad 1339(\mathrm{CH}), 1317\left(\mathrm{~N}-\mathrm{H}+\mathrm{C}-\mathrm{N}_{\text {Amid III }}, \mathrm{CH}\right)$, 1260 (C-O-C, OH, C-N), 1244 (C-O-C, C-OH), 1149 (C-O-C, C-OH), 1057 (C-O-C, C-OH), 1015 (C-O-C, $\mathrm{C}-\mathrm{OH}$ ), 995 (C-O-C, C-OH, C-C), $889(\mathrm{CH}), 720\left(\mathrm{NH}_{\text {Amid }}\right.$ ), $679\left(\mathrm{NH}_{2}\right), 661(\mathrm{OH}), 635(\mathrm{CH}), 617\left(\mathrm{O}=\mathrm{C}-\mathrm{N}_{\text {Amid IV }}\right)$, $603(\mathrm{CH}), 567(\mathrm{NH}, \mathrm{CO})$.

IR spectrum of composite $1\left(\mathrm{v}, \mathrm{cm}^{-1}\right): 3297(\mathrm{OH}, \mathrm{NH})$, $2989(\mathrm{CH}), 2948(\mathrm{CH}), 2888(\mathrm{CH}), 2836 \quad(\mathrm{CH})$, 1731 (C=Oрмmа $), \quad 1715 \quad$ (C=O = $1636\left(\mathrm{NH}_{2}\right), 1623\left(\mathrm{NH}_{2}\right), 1610\left(\mathrm{NH}_{2}\right), 1558\left(\mathrm{NH}_{2}\right)$, $1542 \quad\left(\mathrm{~N}-\mathrm{H}+\mathrm{C}-\mathrm{N}_{\text {Amid II }}\right), \quad 1517 \quad\left(\mathrm{~N}-\mathrm{H}+\mathrm{C}-\mathrm{N}_{\text {Amid II }}\right)$, $1508\left(\mathrm{~N}-\mathrm{H}+\mathrm{C}-\mathrm{N}_{\text {Amid II }}\right), 1488(\mathrm{CH}), 1474(\mathrm{CH}), 1451(\mathrm{CH})$, $1433(\mathrm{CH}), 1418(\mathrm{CH}), 1397(\mathrm{CH}), 1387(\mathrm{CH}), 1379(\mathrm{CH})$, $1363(\mathrm{CH}), 1339(\mathrm{CH}), 1318\left(\mathrm{~N}-\mathrm{H}+\mathrm{C}-\mathrm{N}_{\text {Amid III }}, \mathrm{CH}\right)$, $1296(\mathrm{CH}), 1268$ (C-O-C, OH, C-N), 1239 (C-O-C, C-OH), $1188\left(\mathrm{CO}-\mathrm{O}-\mathrm{CH}_{3}, \mathrm{CH}\right), 1145$ (C-O-C, C-OH), 1086 (C-O-C, $\mathrm{C}-\mathrm{OH}), 106 \mathrm{O}$ (C-O-C, C-OH), 1034 (C-O-C, C-OH), 1012 (C-O-C, C-OH), 991 (C-O-C, C-OH, C-C), 966 (C-C), $943(\mathrm{CH}), 838(\mathrm{CH}), 812(\mathrm{C}=\mathrm{O}), 747(\mathrm{C}=\mathrm{O}, \mathrm{CH})$, $720\left(\mathrm{NH}_{\text {Amid V }}\right), 686(\mathrm{CH}), 679\left(\mathrm{NH}_{2}\right), 66 \mathrm{o}(\mathrm{OH}), 636(\mathrm{CH})$, $618\left(\mathrm{O}=\mathrm{C}-\mathrm{N}_{\text {Amid IV }}\right), 6 \mathrm{O} 2(\mathrm{CH}), 572(\mathrm{CH}), 558(\mathrm{NH}, \mathrm{CO})$.

IR spectrum of composite $2\left(\mathrm{v}, \mathrm{cm}^{-1}\right): 3247(\mathrm{OH}, \mathrm{NH})$, $2996(\mathrm{CH}), 2947(\mathrm{CH}), 2865(\mathrm{CH}), 1732$ (C=O

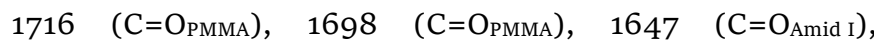
$1636\left(\mathrm{NH}_{2}\right), 1623\left(\mathrm{NH}_{2}\right), 1617 \quad\left(\mathrm{NH}_{2}\right), 1609\left(\mathrm{NH}_{2}\right)$, $1557\left(\mathrm{NH}_{2}\right), 1541\left(\mathrm{~N}-\mathrm{H}+\mathrm{C}-\mathrm{N}_{\text {Amid II }}\right), 1522\left(\mathrm{~N}-\mathrm{H}+\mathrm{C}-\mathrm{N}_{\text {Amid II }}\right)$,
$1507\left(\mathrm{~N}-\mathrm{H}+\mathrm{C}-\mathrm{N}_{\text {Amid II }}\right), 1497\left(\mathrm{~N}-\mathrm{H}+\mathrm{C}-\mathrm{N}_{\text {Amid II }}\right), 1489(\mathrm{CH})$, $1473(\mathrm{CH}), 1456(\mathrm{CH}), 1435(\mathrm{CH}), 1419(\mathrm{CH}), 1396(\mathrm{CH})$, $1387(\mathrm{CH}), \quad 1374(\mathrm{CH}), 1362(\mathrm{CH}), 1339 \quad(\mathrm{CH})$, 1319 (N-H+C-N Amid III, CH), 1268 (C-O-C, OH, C-N), 1241 (C-O-C, C-OH), $1186\left(\mathrm{CO}-\mathrm{O}-\mathrm{CH}_{3}, \mathrm{CH}\right), 1141$ (C-O-C, $\mathrm{C}-\mathrm{OH}), 1090$ (C-O-C, C-OH), $106 \mathrm{O}$ (C-O-C, C-OH), 1028 (C-O-C, C-OH), 1012 (C-O-C, C-OH), 985 (C-O-C, $\mathrm{C}-\mathrm{OH}, \mathrm{C}-\mathrm{C}), 968(\mathrm{C}-\mathrm{C}), 905(\mathrm{CH}), 839(\mathrm{CH}), 807(\mathrm{C}=\mathrm{O})$, $747(\mathrm{C}=\mathrm{O}, \mathrm{CH}), 720\left(\mathrm{NH}_{\text {Amid }}\right), 696(\mathrm{CH}), 681\left(\mathrm{NH}_{2}\right)$, $650(\mathrm{OH}), 617\left(\mathrm{O}=\mathrm{C}-\mathrm{N}_{\text {Amid IV }}\right), 601(\mathrm{CH}), 572(\mathrm{CH})$, $562(\mathrm{NH}, \mathrm{CO})$.

IR spectrum of composite $3\left(\mathrm{v}, \mathrm{cm}^{-1}\right): 3239(\mathrm{OH}, \mathrm{NH})$, $3031(\mathrm{CH}), 2993(\mathrm{CH}), 2951(\mathrm{CH}), 2801 \quad(\mathrm{CH})$, 1720 (C=Oрмма $), 1655$ (C=OAmid I $), 1638\left(\mathrm{NH}_{2}\right), 1627\left(\mathrm{NH}_{2}\right)$, $1619\left(\mathrm{NH}_{2}\right), 1610\left(\mathrm{NH}_{2}\right), 1561\left(\mathrm{NH}_{2}\right), 1542(\mathrm{~N}-\mathrm{H}+\mathrm{C}-\mathrm{N}$ Amid II $)$, $1523 \quad\left(\mathrm{~N}-\mathrm{H}+\mathrm{C}-\mathrm{N}_{\text {Amid II }}\right), \quad 1509 \quad\left(\mathrm{~N}-\mathrm{H}+\mathrm{C}-\mathrm{N}_{\text {Amid II }}\right)$, $1499\left(\mathrm{~N}-\mathrm{H}+\mathrm{C}-\mathrm{N}_{\text {Amid II }}\right), 1474(\mathrm{CH}), 1458(\mathrm{CH}), 1449(\mathrm{CH})$, $1432(\mathrm{CH}), 1419(\mathrm{CH}), 1396(\mathrm{CH}), 1387(\mathrm{CH}), 1376(\mathrm{CH})$, $1365(\mathrm{CH}), 1338(\mathrm{CH}), 1323\left(\mathrm{~N}-\mathrm{H}+\mathrm{C}-\mathrm{N}_{\text {Amid III }}, \mathrm{CH}\right)$, $1297(\mathrm{CH}), 1267(\mathrm{C}-\mathrm{O}-\mathrm{C}, \mathrm{OH}, \mathrm{C}-\mathrm{N}), 1239(\mathrm{C}-\mathrm{O}-\mathrm{C}, \mathrm{C}-\mathrm{OH})$, $1185\left(\mathrm{CO}-\mathrm{O}-\mathrm{CH}_{3}, \mathrm{CH}\right), 1139(\mathrm{C}-\mathrm{O}-\mathrm{C}, \mathrm{C}-\mathrm{OH}), 1102(\mathrm{C}-\mathrm{O}-\mathrm{C}$, $\mathrm{C}-\mathrm{OH}), 1082$ (C-O-C, C-OH), 1062 (C-O-C, C-OH), 1029 (C-O-C, C-OH), 1016 (C-O-C, C-OH), 987 (C-O-C, $\mathrm{C}-\mathrm{OH}, \mathrm{C}-\mathrm{C}), 961(\mathrm{C}-\mathrm{C}), 917(\mathrm{CH}), 838(\mathrm{CH}), 814(\mathrm{C}=\mathrm{O})$, $798(\mathrm{CH}), 780(\mathrm{CH}), 747(\mathrm{C}=\mathrm{O}, \mathrm{CH}), 723\left(\mathrm{NH}_{\text {Amid } \mathrm{V}}\right)$, $694(\mathrm{CH}), 684\left(\mathrm{NH}_{2}\right), 654(\mathrm{OH}), 620\left(\mathrm{O}=\mathrm{C}-\mathrm{N}_{\mathrm{Amid}} \mathrm{IV}\right), 607(\mathrm{CH})$, $573(\mathrm{CH}), 561(\mathrm{NH}, \mathrm{CO})$.

\section{Results and discussion}

Composites of chitosan and PMMA were obtained by in situ polymerization with heating at different temperatures (Fig. 1). To obtain composite 3, mechanochemical activation was preliminarily carried out by grinding chitosan and PMMA powders for $120 \mathrm{~min}$. The source of PMMA was the Villacryl H Plus heat-curing acrylic resin for denture bases [6].

The resulting composites were analyzed by ATR FT-IR spectroscopy. It is often used to research composite materials [10]. a

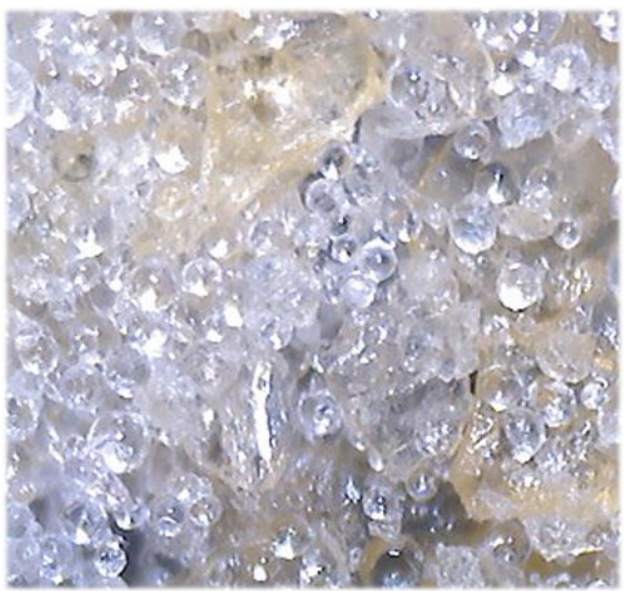

$\mathrm{b}$

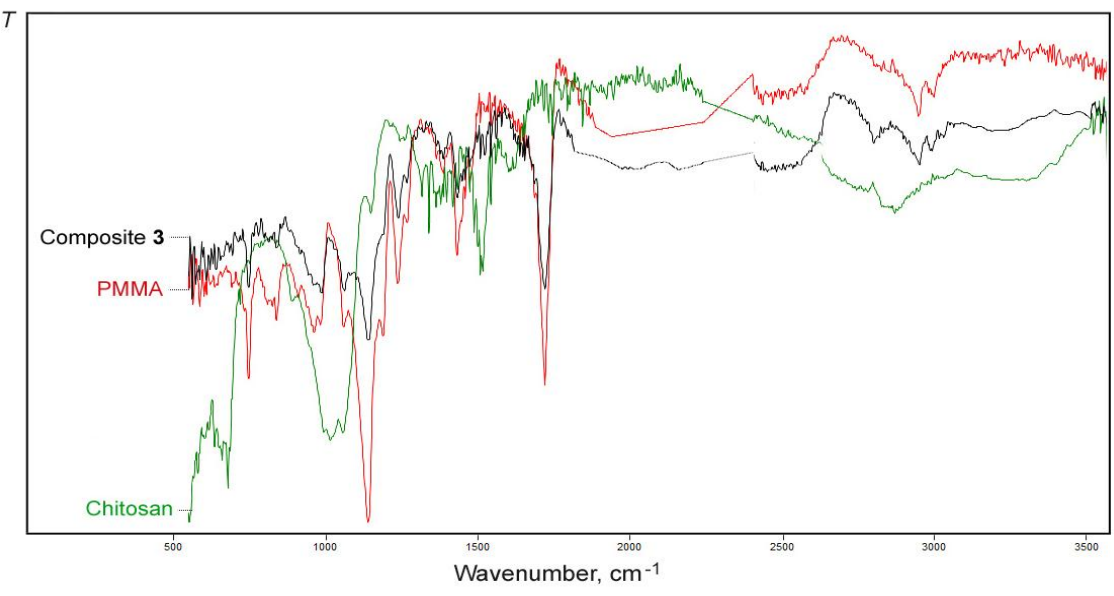

Fig. 1 Photo of the PMMA-chitosan composite 3 (a) and ATR FT-IR spectra of PMMA, chitosan and PMMA-hitosan composite 3 (b) 
The IR spectra of chitosan and PMMA composites show low-frequency shifts of the absorption band of stretching vibrations of $\mathrm{O}-\mathrm{H}$ and $\mathrm{N}-\mathrm{H}$ bonds in chitosan from $3310 \mathrm{~cm}^{-1}$ to $3297 \mathrm{~cm}^{-1}$ (for composite 1 ), to $3247 \mathrm{~cm}^{-1}$ (for composite 2) and to $3239 \mathrm{~cm}^{-1}$ (for composite 3; Fig. 1). Such changes in the spectra confirm the formation of hydrogen bonds.

In addition, IR spectra of composites 1-3 show certain changes related to the absorption bands of stretching vibrations of $\mathrm{C}-\mathrm{O}$ bonds in $\mathrm{C}-\mathrm{O}-\mathrm{C}$ and $\mathrm{C}-\mathrm{OH}$ groups, as well as to the in-plane bending vibrations of $\mathrm{O}-\mathrm{H}$ bonds in chitosan: $1260 \rightarrow 1268,1244 \rightarrow 1239,1149 \rightarrow 1145,1057 \rightarrow 1060$, $1015 \rightarrow 1012$, 995 $\rightarrow 991 \mathrm{~cm}^{-1}$ (for composite 1 ), $1260 \rightarrow 1268$, $1244 \rightarrow 1241, \quad 1149 \rightarrow 1141, \quad 1057 \rightarrow 1060, \quad 1015 \rightarrow 1012$, $995 \rightarrow 985 \mathrm{~cm}^{-1}$ (for composite 2 ), and $1260 \rightarrow 1267$, $1244 \rightarrow 1239$, 1149 $\rightarrow 1139$, 1057 $\rightarrow 1062$, 995 $\rightarrow 987 \mathrm{~cm}^{-1}$ (for composite 3 ). In this case, the most significant shifts were noted for the composites 2 and 3 .

The main absorption band of $\mathrm{C}=\mathrm{O}$ stretching vibrations in PMMA ester bond was found at $1715 \mathrm{~cm}^{-1}$ (composite 1), at $1716 \mathrm{~cm}^{-1}$ (composite 2) and at $1720 \mathrm{~cm}^{-1}$ (composite 3 ). Moreover, additional bands are observed at 1698 and $1732 \mathrm{~cm}^{-1}$ (for composite 1), and at $1731 \mathrm{~cm}^{-1}$ (for composite 2). The presence of low-frequency shifts in the IR spectra is indicative of involving $\mathrm{C}=\mathrm{O}$ group of PMMA in hydrogen bonding with chitosan.

High-frequency shifts of some absorption bands of inplane bending vibrations of $\mathrm{N}-\mathrm{H}$ bonds in the chitosan molecule are observed in the spectra of composites: $1602 \rightarrow 1610 \mathrm{~cm}^{-1}$ (for composite 1 ), $1602 \rightarrow 1609 \mathrm{~cm}^{-1}$ (for composite 2 ), $1623 \rightarrow 1627 \mathrm{~cm}^{-1}$ (for composite 3 ). In addition, the IR spectra show shifts of the main absorption bands of stretching vibrations of $\mathrm{CH}$ bonds, which can be caused by hydrophobic interactions during the formation of composites.

\section{Conclusions}

Polymer composites of PMMA and chitosan were obtained by polymerization with heating and mechanochemical method. The composites 1-3 are formed due to hydrogen bonds ( $\mathrm{C}=$ Opmma...H-Ochitosan and $\mathrm{C}=\mathrm{O}$ Pmma...H-NHchitosan) and hydrophobic interactions. It is possible that the presence of chitosan in composite materials can change some of their mechanical properties and eliminate the toxicity of PMMA.

\section{Acknowledgements}

This study was carried out on the experimental equipment of the Sevastopol State University (project PR/80742/2017).

\section{References}

1. Stein PS, Sullivan J, Haubenreich JE, Osborne PB. Composite resin in medicine and dentistry. J Long Term Eff Med Implants. 2005;15(6):641-654. doi:10.1615/jlongtermeffmedimplants.v15.i6.70

2. Scharf F, Mikhnevich E, Safronov A. Interaction of iron oxide nanoparticles synthesized by laser target evaporation with polyacrylamide in composites and ferrogels. Chimica Techno Acta. 2017;4(2):128-139. doi:10.15826/chimtech/2017.4.2.028

3. Mikhnevich EA, Safronov AP, Beketov IV, Medvedev AI. Carbon coated nickel nanoparticles in polyacrylamide ferrogels: interaction with polymeric network and impact on swelling. Chimica Techno Acta. 2020;7(3):116-127. doi:10.15826/chimtech.2020.7.3.04

4. Frazer RQ, Byron RT, Osborne PB, West KP. PMMA: an essential material in medicine and dentistry. J Long Term Eff Med Implants. 2005;15(6):629-639. doi:10.1615/jlongtermeffmedimplants.v15.i6.60

5. Liber-Kneć A, Łagan S. Surface testing of dental biomaterialsdetermination of contact angle and surface free energy. Materials (Basel). 2021;14(11):2716. doi:10.3390/ma14112716

6. Solutions for the dental laboratory. Catalogue [Internet]. Zhermack S.p.A., 2019. p. 72-79. Available from: https://www.zhermack.com/public/uploads/F300072_19o7 Catalogo LABO EN low.pdf

7. Leggat PA, Kedjarune U. Toxicity of methyl methacrylate in dentistry. Int Dent J. 2003;53(3):126-131. doi:10.1111/j.1875-595x.2003.tboo736.x

8. Zargar V, Asghari M, Dashti A. A review on chitin and chitosan polymers: structure, chemistry, solubility, derivatives and applications. Chem Bio Eng Rev. 2015;2(3):204-226. doi:10.1002/cben.201400025

9. Kusmono, Abdurrahim I. Water sorption, antimicrobial activity, and thermal and mechanical properties of chitosan/clay/glycerol nanocomposite films. Heliyon. 2019;5(8):eo2342. doi:10.1016/j.heliyon.2019.eo2342

10. Voron'ko N, Sokolan N, Kuchina Y, Berestova G. Formation of polyelectrolyte complexes from chitosan and alkaline gelatin. KnE Life Sciences. 2020;5(1):109-119. doi:10.18502/kls.v5i1.6031

11. Smirnova NN, Markin AV, Smirnova ON, Smirnov VF, Knyazev AV. Effect of technogenic factors and biodestructive agents on the thermal behavior of chitosan and poly(methyl acrylate) block copolymer. Russ J Phys Chem. 2020;94(6):1262-1267. doi:10.1134/So036024420060254 